\title{
Acupuncture Techniques and Devices
}

\author{
LIXING LAO, Ph.D., L.Ac.
}

\section{INTRODUCTION}

D uring the long history of Chinese medicine, acupuncture developed along with other types of ancient Chinese medicine such as herbs, Chinese massage (Tui Na), diet therapy, and mind/body exercise (e.g., Qi Gong). The original definition of acupuncture (Zheng Jiu) means needling and moxibustion. Acupuncture sometimes also includes cupping techniques. The acupuncture devices used in modern society are far more advanced in terms of quality, compared with what was used thousands of years ago.

\section{Needling}

Acupuncture needles have been believed to be in use about 4,000 years. The earliest acupuncture instruments found in archaeological findings in China were made out of stone and were called bian (1700 B.C.). Through the history of human development, the materials of the needles have varied from ceramic, bamboo, bronze, iron, gold, and silver. Presently, the most commonly used needles are made from stainless steel, and the quality of these needles has greatly improved.

The needles were described as sharp, dull, long, or short, and were first mentioned as the "Nine Needles" in the ancient classic book Huang Di Nei Jing (The Yellow Emperor's Inner Classic, 2nd century B.C.). The purpose of the Nine Needles was for insertion into the acupuncture points or for pressure on the sur- face of points on the skin. It was also used for surgical procedures or for an acupuncture bloodletting technique. The function of needling is believed to be to promote Qi (the vital energy) in the meridians in order to produce its therapeutic effect.

\section{Moxibustion}

Moxibustion is a method in which a moxa herb (Artemisia vulgaris) is burned above the skin or on the acupuncture points. It can be used as a cone, stick, loose herb, or applied at the end of the acupuncture needles. The purpose of moxibustion is to apply heat to the acupuncture points to alleviate symptoms.

\section{Cupping}

Cupping was known as the "horn method" because the original cups were made out of the horn of animals. Various materials have been used for cupping such as bamboo, glass, and ceramics. The purpose of cupping is to create a vacuum or negative pressure on the surface of the skin. This promotes blood circulation and stimulates the acupuncture point.

\section{DEVELOPMENT OF ACUPUNCTURE TECHNIQUES AND DEVICES}

\section{Acupuncture Techniques and Devices}

Generally, there are two different types of acupuncture devices currently being used 
worldwide (mainly in China). One set of devices is derived from the original classical Nine Needles. These include filiform needles, cutaneous needles, pressure needles, intradermal needles, "beard of wheat" needles, "barefoot doctor" needles, great needles, pyramid needles (bloodletting), warm needle acupuncture, hot needle acupuncture, and surgical acupuncture. The other set combines classical acupuncture devices with modern technology such as electroacupuncture, injection acupuncture, laser acupuncture, ultraviolet acupuncture, and magnetic acupuncture. These techniques have been developed within the past several decades. In the United States, acupuncture devices such as filform needles, intradermal needles (auricular acupuncture), electroacupuncture, and laser acupuncture are commonly used.

\section{Acupuncture Forms}

In addition to classical body acupuncture, several forms of acupuncture have been developed in terms of the location of stimulation. These types of acupuncture include auricular acupuncture, scalp acupuncture, face acupuncture, hand acupuncture, nose acupuncture, foot acupuncture, and trigger point acupuncture. Among these forms, auricular acupuncture has been most commonly used.

\section{Acupuncture Styles (Schools)}

Since acupuncture has spread from its basic origin across the world, different acupuncture styles (schools) have been developed. In the United States, there are several acupuncture styles that are commonly practiced such as traditional Chinese medical acupuncture, Japanese acupuncture (e.g., meridian therapy), English acupuncture (e.g., traditional acupuncture), French acupuncture (e.g., French and American medical acupuncture), Korean acupuncture (e.g., constitutional acupuncture), and others. Most of these types of acupuncture are derived from ancient Chinese medical philosophy and practices.

\section{FILIFORM ACUPUNCTURE NEEDLE}

\section{Manufacturers}

The major sources of acupuncture needles in the American market are from China, Japan, and Korea. There are a number of distributors in the United States such as OMS Medical Supplies, TCM Supply Corporation, Acupuncture Supplies Import, Dong Bank U.S.A., CAI industries, Lhasa Medical, Scientific Health Care, and Eastern Currents. The range of prices of filiform needles is $\$ 0.05-0.14$ each.

\section{Quality and Technique Parameters}

The manufacturing of modern acupuncture needles is adapted from hypodermal and suturing needles (Class I, 878.8400), with additional technical criteria to meet the requirements of acupuncture needle usage. Usually, acupuncture needles stay in the human body for a period of time and are often manipulated by the practitioner.

Structure and Size. Acupuncture needles usually consists of five parts: (1) the handle, (2) the tail (end of the handle), (3) the tip, (4) the body, (5) and the root (connection between the body and the handle). The size of the needle is based on the diameter and length of the needle body. Needle body diameters range from $0.12-0.4$ $\mathrm{mm}$ (commonly $0.20-0.28 \mathrm{~mm}$ ) and have lengths from 7-200 mm (commonly 15-60 mm).

Material. The material of the needle body is stainless steel (0Cr18Ni9 or $1 \mathrm{Cr} 18 \mathrm{Ni9}$ ), which is equivalent to Class 3, Austenitic Stainless Steel Type 302, 304, or 316 of ASTM F-899-84. The handle is made from either stainless steel, polypropylene plastic, copper wire with silver plating, or bronze.

Strength. The penetration resistance of the needle is $5-10-\mathrm{g}$ force. The needle tensile strength is over $2-\mathrm{kg}$ force or $250 \mathrm{kpsi}$ minimum.

Sterile Procedure. Sterilization of the disposable needles is done with either ethylene oxide gas (E.T.O.) or with radiation (2.5 Mrad gamma rays).

Package. Disposable needles are packed either individually or in bulk packages of 2, 5 , 10,20 , or 50 needles per package in microbial barrier medical packaging paper and plastic sheathing.

Labeling. The packaging box meets the Federal Drug and Food Administration (FDA) requirement specified by $C F R-801$ and contains 
the name of the product, the name of the manufacturer, the lot number, the size of the needles, the number of the needles, how the needles were packaged (e.g., individually packed needle with tube), the storage condition, the method of sterilization (e.g., E.T.O.), the date of sterilization, the date of expiration, and the FDA-required caution of usage. Some products indicate the FDA registration number.

\section{Needling (Filiform) Techniques}

The technique for filiform needles mainly refers to body acupuncture. Skin preparation and needle insertion should follow standard procedure (e.g., Clean Needle Technique for Acupuncturists: A Manual). ${ }^{2}$ Generally, after needle insertion, there are three angles formed between the needle and the surface of the skin: perpendicular $\left(90^{\circ}\right)$, oblique $\left(45^{\circ}\right)$, and horizontal $\left(15^{\circ}\right)$. The angles and depths are based on the anatomical location of the point, the therapeutic purpose, and the size of the patient.

For treatment purposes, most acupuncturists manually manipulate the needle after insertion. The basic method of manipulation includes lifting and thrusting (moving the needle in the vertical direction), twirling and rotating (usually up to $360^{\circ}$ ), or a combination of the two. These methods are used to obtain the 'De Qi' sensation, which is the feeling of heaviness, numbness, or tightness by the patient; and the operator should feel tenseness around the needle. It is thought to be an important validation of the acupuncture effect as described in acupuncture textbooks.

Acupuncture treatment is based on (1) Western diagnosis, (2) Oriental diagnosis, (3) or a combination of both. The acupuncture point location depends on the type of training the acupuncturist has received (e.g., Chinese eightprinciple acupuncture, English five-element acupuncture, Japanese meridian acupuncture, trigger-point acupuncture). The selection of the acupuncture points can be based on the condition of the individual patient or a formula of a set of fixed acupuncture points used for specific symptoms. The average number of needles that are used range from 4 to 20 needles per treatment. The needles are left in place from approximately a few seconds to 30 minutes, and in some instances, the needles may be left in place for a longer period of time.

\section{REFERENCES}

1. Cheng $X$, ed. Chinese Acupuncture and Moxibustion (1st ed.). Beijing: Foreign Languages Press, 1987.

2. Davis E, Howard M, McCormack J, eds. Clean Needle Technique for Acupuncturist: A Manual. Washington DC: National Commission for the Acupuncturist, 1989.

3. Jiang XS. Acupuncture Needles (National Standard of the People's Republic of China). Beijing: China Standard, 1988.

4. Kaptchuk TJ. The Web That Has No Weaver. New York: Congdon \& Weed, 1983.

5. Lytle CD. An Overview of Acupuncture. DHHS, PHS, FDA, Washington, DC: U.S. GPO. 1993.

6. O'Conner J, Bensky D. Acupuncture: A Comprehensive Text. Chicago: Eastland Press, 1981.

7. Stux G, Pomeranz B. History of acupuncture. In: Acupuncture: Textbook and Atlas. Berlin: SpringerVerlag, 1987.

Address reprint requests to: Lixing Lao, Ph.D., L.Ac. Research Assistant Professor Division of Complementary Medicine Kernan Hospital Mansion 2200 N. Forest Park Avenue Baltimore, MD 21207-6697 


\section{This article has been cited by:}

1. D. T. W. Yu, A. Y. M. Jones. 2013. Physiological changes associated with de qi during electroacupuncture to LI4 and LI11: a randomised, placebo-controlled trial. Acupuncture in Medicine 31:2, 143-150. [CrossRef]

2. Dan Marlowe. 2012. Complementary and Alternative Medicine Treatments for Low Back Pain. Primary Care: Clinics in Office Practice . [CrossRef]

3. Ping-Chung Leung, Ellie S.Y. Pang. 2011. Rising Popularity of Acupuncture Treatment: Justifications, Clinical Research, and Difficulties. Medical Acupuncture 23:3, 143-149. [Abstract] [Full Text HTML] [Full Text PDF] [Full Text PDF with Links]

4. Andrea D Furlan, Fatemeh Yazdi, Alexander Tsertsvadze, Lixing Lao, Karen Sherman, Carlo Ammendolia, Ellen Wang, ByungCheul Shin, Ikuro Wakayama, Maurits W van Tulder, Andrea D FurlanAcupuncture for (sub)acute non-specific low-back pain . [CrossRef]

5. W. Zukow, Z. Kalisz, R. Muszkieta, M. Napierala. 2011. Acupuncture for rheumatoid arthritis: A randomized, sham-controlled clinical trial. Journal of Acupuncture and Tuina Science 9:3, 168-172. [CrossRef]

6. Mitchell K. Freedman, Madhuri Dholakia, Dennis W. Ivill, Alan S. Hilibrand, Zach BroyerCervical Radiculopathy 713-722. [CrossRef]

7. Petros Efthimiou, Manil Kukar. 2010. Complementary and alternative medicine use in rheumatoid arthritis: proposed mechanism of action and efficacy of commonly used modalities. Rheumatology International 30:5, 571-586. [CrossRef]

8. Eunice K. Tan, George W. M. Millington, Nick J. Levell. 2009. Acupuncture in dermatology: an historical perspective. International Journal of Dermatology 48:6, 648-652. [CrossRef]

9. Yanhua Deng, Xiaojing Ma, Weina Peng, Lara Sanvito, Yanhua DengAcupuncture for Guillain-Barré syndrome . [CrossRef]

10. C AMMENDOLIA, A FURLAN, M IMAMURA, E IRVIN, M VANTULDER. 2008. Evidence-informed management of chronic low back pain with needle acupuncture. The Spine Journal 8:1, 160-172. [CrossRef]

11. S SAKAI, E HORI, K UMENO, N KITABAYASHI, T ONO, H NISHIJO. 2007. Specific acupuncture sensation correlates with EEGs and autonomic changes in human subjects. Autonomic Neuroscience 133:2, 158-169. [CrossRef]

12. Mike Cummings, Peter Baldry. 2007. Regional myofascial pain: diagnosis and management. Best Practice \& Research Clinical Rheumatology 21:2, 367-387. [CrossRef]

13. Kien Trinh, Nadine Graham, Anita Gross, Charlie Goldsmith, Ellen Wang, Ian Cameron, Theresa Kay. 2007. Acupuncture for Neck Disorders. Spine 32:2, 236-243. [CrossRef]

14. Wei Kou, Isabel Gareus, John D. Bell, Marion U. Goebel, Günther Spahn, Gustavo Pacheco-López, Marcus Bäcker, Manfred Schedlowski, Gustav J. Dobos. 2007. Quantification of DeQi Sensation by Visual Analog Scales in Healthy Humans after Immunostimulating Acupuncture Treatment. The American Journal of Chinese Medicine 35:05, 753-765. [CrossRef]

15. Kien Trinh, Nadine Graham, Anita Gross, Charles H Goldsmith, Ellen Wang, Ian D Cameron, Theresa M Kay, Kien TrinhAcupuncture for neck disorders . [CrossRef]

16. Baixiao Zhao, Xinjuan Wang, Zhixiu Lin, Renquan Liu, Lixing Lao. 2006. A novel sham moxibustion device: A randomized, placebo-controlled trial. Complementary Therapies in Medicine 14:1, 53-60. [CrossRef]

17. M VANTULDER, A FURLAN, J GAGNIER. 2005. Complementary and alternative therapies for low back pain. Best Practice \& Research Clinical Rheumatology 19:4, 639-654. [CrossRef]

18. Andrea D. Furlan, Maurits van Tulder, Dan Cherkin, Hiroshi Tsukayama, Lixing Lao, Bart Koes, Brian Berman. 2005. Acupuncture and Dry-Needling for Low Back Pain: An Updated Systematic Review Within the Framework of the Cochrane Collaboration. Spine 30:8, 944-963. [CrossRef]

19. Andrea D Furlan, Maurits W van Tulder, Dan Cherkin, Hiroshi Tsukayama, Lixing Lao, Bart W Koes, Brian M Berman, Andrea D FurlanAcupuncture and dry-needling for low back pain . [CrossRef]

20. KV Trinh, TM Kay, N Graham, AR Gross, CH Goldsmith, I Cameron, E Wang, Z Radylovic, Kien TrinhAcupuncture for neck disorders . [CrossRef]

21. Maurits W. van Tulder, Daniel C. Cherkin, Brian Berman, Lixing Lao, Bart W. Koes. 1999. The Effectiveness of Acupuncture in the Management of Acute and Chronic Low Back Pain. Spine 24:11, 1113. [CrossRef]

22. MW van Tulder, DC Cherkin, B Berman, L Lao, BW KoesAcupuncture for low-back pain . [CrossRef]

23. David M. Schuster. 1996. The Integrative Hospital Explored via Acupuncture. The Journal of Alternative and Complementary Medicine 2:4, 503-514. [Abstract] [Full Text PDF] [Full Text PDF with Links] 